\title{
Association between ADIPOQ Gene Polymorphism rs182052 and Obesity in Korean Women
}

\author{
Miae Doo and Yangha Kim* \\ Department of Nutritional Science and Food Management, \\ Ewha Womans University, Seoul 120-750, Korea
}

\begin{abstract}
The association between adiponectin concentration and obesity have been reported and genetic variations of the ADIPOQ gene are known to influence the plasmatic concentration of adiponectin. Therefore, we investigated the effect of AIPOQ single nucleotide polymorphism (SNP) on obesity-related variables, and their modulation by dietary intakes in Korean women. The subjects consisted of 3,217 Korean women aged 40-59 years participating in the Korean Genome Epidemiology Study (KoGES). The general characteristics, anthropometric variables, serum blood profiles were measured. Dietary intake was analyzed using the Food Frequency Questionnaire. Subjects with the T allele of AIPOQ rs182052 showed significantly higher obesity-related variables such as weight $(p=0.005)$, BMl $(p<0.000)$, fat body mass $(p=0.005)$, and waist-hip ratio $(p=0.007)$ than those with the $\mathrm{C}$ allele. Moreover, the rs182052 T allele was associated with an increased risk of obesity prevalence $(p=0.019)$. However, there were not any significant interactions observed between the genotype of ADIPOQ rs182052 and dietary intake on BMI and fat body mass. These findings suggest that the obesity-related variables may be more dominantly affected by the genotype of ADIPOQ rs182052 than dietary intake in middle aged Korean women.
\end{abstract}

Keywords: ADIPOQ, single nucleotide polymorphism, rs182052, obesity, gene-diet interactions

\section{Introduction}

The figure of obese people worldwide was at least 400 million in 2005; this figure is predicted to rise to approximately 2.3 billion by 2015 (WHO, 2006). The primary cause of obesity is known to be an accumulation of excess body fat occurring through an imbalance of energy

*Corresponding author: E-mail yhmoon@ewha.ac.kr Tel +82-2-3277-3101, Fax +82-2-3277-2862 Accepted 1 September 2010 intake over energy expenditure (Walker et al., 2007). However, it has been reported that genetic variations may influence individuals to show different predispositions to obesity (Fawcett et al., 2010; Loos, 2009). ADIPOQ, the gene coding for adiponectin, is located on chromosome $3 \mathrm{q} 27$ and is expressed in adipose tissue exclusively. Several single nucleotide polymorphisms (SNPs) in the ADIPOQ gene are known to influence the plasmatic concentration of the encoded protein. These SNPs have shown a linkage to obesity as well as type 2 diabetes, metabolic syndrome, cholesterol concentration and coronary artery disease (Heid et al., 2010; Hivert et al., 2008; Kadowak et al., 2006; Li et al., 2009; Ntall et al., 2009). In particular, the genetic variations of rs 182052 which is located in exon 1 of the ADIPOQ gene, have been reported to be associated with obesity-related variables (Richardson et al., 2006; Wassel et al., 2010).

Nowadays, it has been reported that an association exists between genetic variation and obesity, and the genetic susceptibility of obesity may be influenced by one's dietary intake (Drewnowski, 2009; Swithers et al., 2010). For instance, the associations of SNPs of the ADIPOQ with BMI and obesity risk were modified by dietary monounsaturated fatty acid intake (Warodomwichit et al., 2009). In a similar manner, the interaction of dietary fiber content and SNP in the ADPOQ could have an influence on childhood obesity (Ntalla et al., 2009).

The aim of the present study was to examine the association between SNP in AIPOQ rs182052 with obesity-related variables such as weight, BMI, fat body mass, and waist-hip ratio, and its modification by dietary intake in middle aged Korean women who participated in the Korean Genome Epidemiology Study (KoGES).

\section{Methods}

\section{Subjects}

The study subjects consisted of 3,217 Korean women aged between 40 to 59 years who participated in the Korean Genome Epidemiology Study (KoGES). The KoGES were performed as cohorts for chronic disease (diabetes, hypertension, osteoporosis, obesity and metabolic syndrome) in adults aged between 40-69, who were recruited from two community-based epidemiology studies in the rural Ansung and urban Ansan commun- 
ities from 2001 to 2008 . The details of the original cohort have been described elsewhere (Ahn et al., 2007; Cho et al., 2009). All subjects were of Korean ancestry. Among 10,038 subjects in KoGES who were available for completed FFQ data and epidemiological data, the women subjects of this study aged between 40-59 were selected. This study protocol was approved by the institutional Review Board of Ewha Womans University Seoul, Korea.

\section{Methods}

\section{Anthropometric variables and blood pressure}

Body weight, height, and waist circumference were measured using a standardized procedure: height without shoes by an anthropometer, weight in light clothes by a weighting scale, waist circumference over the unclothed abdomen at a minimal diameter by non stretchable standard tape. Body composition (lean body mass, fat body mass, and Waist/Hip ratio) were measured by an In-body 3.0 (Biospace Co., Ltd, Seoul, Korea). Body mass index (BMI) was calculated by dividing the weight in kilograms by height in meters squared. Obesity was defined as the BMI $\geq 25 \mathrm{~kg} / \mathrm{m}^{2}$, which were WHO AsiaPacific Area criteria for obesity (WHO, 2000).

Systolic blood pressure and diastolic blood pressure were measured with the subject in a lying position after 5 minutes of rest. The average number of three blood pressure readings, which were conducted in between intervals of 30 seconds, was used for analysis.

\section{Serum blood profile}

After 8-14 $\mathrm{h}$ of overnight fasting, blood samples were collected to measure plasma glucose, insulin, total cholesterol, HDL-cholesterol, and triglycerides using the Hitachi 7600 Automatic Analyzer (Hitachi, Tokyo, Japan). LDL-Cholesterol levels were calculated by the following equations described by Friedewald for subjects with a level of serum triglycerides $<400 \mathrm{mg} / \mathrm{dl}$ (Friedewald et al., 1972).

LDL-cholesterol $=\quad[$ Total cholesterol- $\{\mathrm{HDL}-$ cholesterol(Triglycerides/5)\}]

\section{Dietary intake}

The usual dietary intake during the previous year was assessed with a Food Frequency Questionnaire (FFQ) for KoGES which has been validated (Ahn et al., 2007). It includes 103 food items and consists of both a 3 portion size and 9 frequency of serving. Dietary intake data were analyzed using Can- Pro 3.0 software (KNS.,
2006). Intake of dietary protein, fat, carbohydrate, and fatty acids (Saturated fatty acid, Monounsaturated fatty acid, and Polyunsaturated fatty acid) were represented as a percentage of the total daily energy intake.

\section{Genotyping and single nucleotide polymorphism selection}

Genomic DNA was extracted from whole blood and genotyped on the Affymetrix Genome-Wide Human SNP array 5.0 [22]. ADOPOQ rs182052, which have been reported to be associated with obesity-related variables in the previous studies (Henneman et al., 2010; Ong et al., 2010; Richardson et al., 2006; Wassel et al., 2010), were selected among SNPs with a minor allele frequency (MAF) $\geq 0.10$ in KoGES GWA study data.

\section{Statistical analysis}

All statistical analyses were performed using SPSS for Windows software (version 17. 0; SPSS Inc., Chicago, IL) with a level of significance at $p<0.05$. Allele frequencies were calculated by allele counting and the departure from Hardy-Weinberg equilibrium was calculated by the Chi-square test. The rs182052 was evaluated with different genetic inherent models and a codominant model was applied in analyses of this study. The data for continuous variables were presented as mean values \pm s.e.m. or $n(\%)$. General linear models after adjustment for potential confounders were applied to compare variables according to the genotype of ADIPOQ rs182052. Also, interactions between the genotype of ADIPOQ rs182052 and dietary intake (as dichotomous) were examined using general linear models after adjustment for potential confounders.

\section{Results and Discussion}

The genotype distribution of SNPs in AIPOQ rs 182052 was in Hardy-Weinberg equilibrium $(p=0.83)$, and the minor allele frequency (MAF) of SNP in AIPOQ rs182052 was 0.49 . This MAF in Korean population observed was different from those in the population of other nations, such as reported figures of 0.40 in European and African populations and 0.47 in Chinese and Japanese populations as obtained from the HapMap data.

In this study, significant associations between obesity-related variables and SNP in ADIPOQ rs182052 were found (Table 1). Subjects with the minor $T$ allele of ADIPOQ rs182052 had greater weight $(p=0.05)$, BMI ( $p$ $<0.000)$, fat body mass $(p=0.005)$, and waist-hip ratio $(p=0.007)$ compared to those with $C$ allele carrier after adjustment for potential confounders. These results cor- 
respond to other studies that showed an association between ADIPOQ rs182052 and BMI (Richardson et al., 2006) or waist circumstance (Wassel et al., 2010). In particular, Richardson et al. (2006) reported that the minor allele of ADIPOQ rs182052 was significantly associated with an increase in BMI, which showed to be in accordance with our results.

In this study, the systolic and diastolic pressures were significantly higher in subjects with minor $\mathrm{T}$ allele of ADIPOQ rs182052 than in those with homozygous C allele carrier after adjustment for potential confounders (Table 1). The genetic variants in the ADIPOQ gene were reported to influence plasma adiponectin level, and one of them showed an association with elevated blood pressures (Henneman et al., 2010; Ong et al., 2010). There were no significant differences in blood profiles according to the genotype of ADIPOQ rs 182052 .

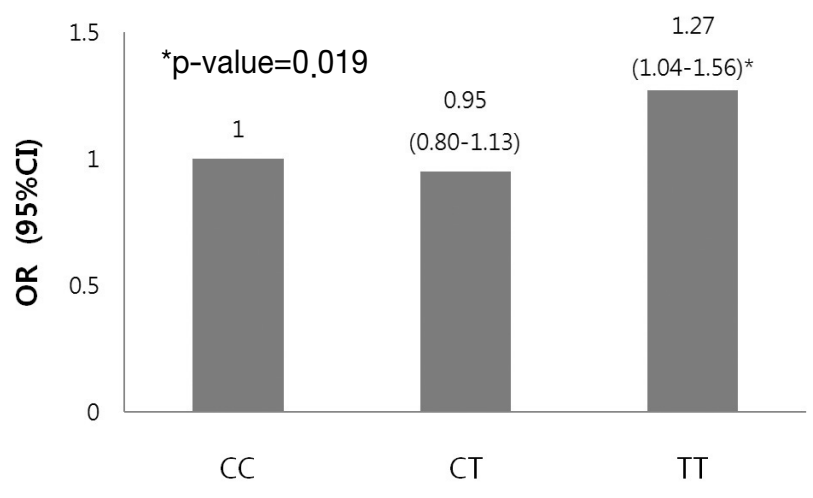

Fig. 1. Odds Ratios (OR) and $95 \%$ confidence interval $(95 \% \mathrm{Cl})$ for obesity prevalence of subject with ADIPOQpolymorphism rs182052.

Table 1. General characteristics of subjects with ADIPOQ polymorphism rs182052

\begin{tabular}{|c|c|c|c|c|}
\hline & CC $(n=839)$ & CT $(n=1,608)$ & $\Pi(\mathrm{n}=770)$ & $p$-value \\
\hline Age (year) & $47.69 \pm 0.19$ & $48.09 \pm 0.14$ & $47.89 \pm 0.20$ & 0.238 \\
\hline Height $(\mathrm{Cm})$ & $154.86 \pm 0.18$ & $155.01 \pm 0.13$ & $154.63 \pm 0.18$ & 0.224 \\
\hline Weight (Kg) & $59.20 \pm 0.28$ & $59.55 \pm 0.21$ & $60.49 \pm 0.30$ & 0.005 \\
\hline $\operatorname{BMI}\left(\mathrm{kg} / \mathrm{m}^{2}\right)$ & $24.68 \pm 0.11$ & $24.78 \pm 0.08$ & $25.29 \pm 0.11$ & $<0.000$ \\
\hline Lean body mass $(\mathrm{kg})$ & $40.51 \pm 0.17$ & $40.60 \pm 0.12$ & $40.90 \pm 0.17$ & 0.228 \\
\hline Fat body mass $(\mathrm{kg})$ & $18.63 \pm 0.20$ & $18.83 \pm 0.14$ & $19.51 \pm 0.21$ & 0.005 \\
\hline Waist-hip ratio & $0.89 \pm 0.00$ & $0.89 \pm 0.00$ & $0.90 \pm 0.00$ & 0.007 \\
\hline Obesity prevalence (\%) & $381(44.0)$ & $699(42.5)$ & $387(48.7)$ & 0.005 \\
\hline \multicolumn{5}{|l|}{ Blood pressure $(\mathrm{mm} \mathrm{Hg})$} \\
\hline Systolic & $112.93 \pm 0.57$ & $112.78 \pm 0.41$ & $115.01 \pm 0.59$ & 0.006 \\
\hline Diastolic & $71.95 \pm 0.40$ & $71.65 \pm 0.29$ & $73.12 \pm 0.41$ & 0.013 \\
\hline Total Cholesterol (mg/dl) & $187.88 \pm 1.16$ & $189.27 \pm 0.84$ & $187.47 \pm 1.21$ & 0.395 \\
\hline HDL-Cholesterol (mg/dl) & $46.37 \pm 0.35$ & $46.16 \pm 0.25$ & $45.78 \pm 0.36$ & 0.489 \\
\hline LDL-Cholesterol (mg/dl) & $113.59 \pm 1.04$ & $115.84 \pm 0.75$ & $114.23 \pm 1.08$ & 0.171 \\
\hline Triglycerides (mg/dl) & $131.73 \pm 2.13$ & $131.99 \pm 1.54$ & $133.42 \pm 2.23$ & 0.833 \\
\hline Glucose (mg/dl) & $83.24 \pm 0.78$ & $83.23 \pm 0.56$ & $83.19 \pm 0.81$ & 0.999 \\
\hline Insulin $(\mu \mathrm{ml})$ & $7.61 \pm 0.15$ & $7.75 \pm 0.11$ & $7.90 \pm 0.15$ & 0.402 \\
\hline
\end{tabular}

Values are mean \pm s.e.m. or $n(\%)$. p-values after adjusted for gender, age, hypertension, diabetes, thyroid gland disease, dyslipidemia, smoking, and alcohol consumption.

Table 2. Dietary intake among subjects with $A D I P O Q$ polymorphism rs182052

\begin{tabular}{lcccc}
\hline & CC $(\mathrm{n}=806)$ & $\mathrm{CT}(\mathrm{n}=1,550)$ & $\pi(\mathrm{n}=743)$ & $\mathrm{p}$-value \\
\hline Energy (kcal) & $1967.34 \pm 19.80$ & $1977.48 \pm 14.28$ & $1967.04 \pm 20.64$ & 0.878 \\
Protein (\% of energy) & $15.71 \pm 0.09$ & $15.75 \pm 0.06$ & $15.71 \pm 0.09$ & 0.901 \\
Fat (\% of energy) & $19.17 \pm 0.19$ & $19.34 \pm 0.14$ & $19.23 \pm 0.20$ & 0.762 \\
Carbohydrate (\% of energy) & $65.78 \pm 0.25$ & $65.60 \pm 0.18$ & $65.74 \pm 0.26$ & 0.82 \\
Saturated fatty acid (\% of energy) & $3.56 \pm 0.06$ & $3.62 \pm 0.04$ & $3.63 \pm 0.06$ & 0.701 \\
Monounsaturated fatty acid (\% of energy) & $3.96 \pm 0.06$ & $4.04 \pm 0.04$ & $3.99 \pm 0.06$ & 0.574 \\
Polyunsaturated fatty acid (\% of energy) & $3.29 \pm 0.04$ & $3.35 \pm 0.03$ & $3.29 \pm 0.04$ & 0.289 \\
\hline
\end{tabular}

Values are mean \pm s.e.m., p-values after adjusted for gender, age, hypertension, diabetes, thyroid gland disease, dyslipidemia, smoking, and alcohol consumption. 
Our data showed that the prevalence of obesity was significantly higher in subjects with the T allele of ADIPOQ rs182052 than in those with homozygous $C$ allele carrier, CC: $44.0 \%$ and $\mathrm{TT}: 48.7 \%$ respectively, after adjustment for potential confounders (Table 1). Moreover, the odds ratio $(\mathrm{OR})$ for obesity prevalence in subjects with ADIPOQ rs182052 T allele was significantly higher compared compared to those with $\mathrm{C}$ allele carrier after adjustment for potential confounders (Fig. 1), implying an increased risk of obesity in subjects with ADIPOQ rs182052 T allele. The hypoadiponectinemia, which was reported to be influenced by genetic variations of the ADIPOQ gene, has been consistently observed in asso- ciation with obesity (Gilardini et al., 2006; Weyer et al., 2001). Therefore, it can be postulated the ADIPOQ rs182052 polymorphism might influence the serum adiponectin, and increase the obesity-related traits and the obesity prevalence.

The dietary intake was analyzed to examine whether genetic variations affect energy intake. No significant differences in dietary intakes of energy, protein, fat, carbohydrate, saturated fatty acid, monounsaturated fatty acid, and polyunsaturated fatty acid were observed among groups with different genotypes of $A D I P O Q$ rs182052 (Table 2). These results suggested that genetic variations in ADIPOQ rs182052 might not influence diet-

Table 3. Effect of interaction between ADIPOQ polymorphism rs 182052 and dietary intake on BMI

\begin{tabular}{lllllcc}
\hline & & CC $(n=806)$ & CT $(n=1,550)$ & TT $(n=743)$ & $p$-value* & $p$-value** \\
\hline Energy, Kcal & Low & $24.71 \pm 0.16$ & $24.75 \pm 0.11$ & $24.12 \pm 0.17$ & 0.123 & 0.576 \\
Protein, \%E & High & $24.66 \pm 0.16$ & $24.77 \pm 0.11$ & $25.39 \pm 0.16$ & 0.002 & \\
Fat, \%E & Low & $24.60 \pm 0.16$ & $24.80 \pm 0.12$ & $25.26 \pm 0.17$ & 0.019 & 0.683 \\
& High & $24.76 \pm 0.16$ & $24.72 \pm 0.11$ & $25.25 \pm 0.16$ & 0.017 & \\
Carbohydrate, \%E & Low & $24.74 \pm 0.16$ & $24.82 \pm 0.12$ & $25.23 \pm 0.17$ & 0.084 & 0.817 \\
Saturated fatty acid, \%E & High & $24.63 \pm 0.15$ & $24.70 \pm 0.11$ & $25.28 \pm 0.16$ & 0.004 & \\
& Low & $24.61 \pm 0.16$ & $24.69 \pm 0.11$ & $25.19 \pm 0.17$ & 0.016 & 0.998 \\
Monounsaturated fatty acid, \%E & High & $24.76 \pm 0.16$ & $24.83 \pm 0.11$ & $25.32 \pm 0.16$ & 0.028 & \\
& Low & $24.82 \pm 0.16$ & $24.85 \pm 0.11$ & $25.51 \pm 0.17$ & 0.002 & 0.510 \\
Polyunsaturated fatty acid, \%E & High & $24.55 \pm 0.16$ & $24.67 \pm 0.12$ & $25.01 \pm 0.16$ & 0.081 & \\
& Low & $24.68 \pm 0.16$ & $24.87 \pm 0.12$ & $25.54 \pm 0.16$ & 0.001 & 0.200 \\
\hline
\end{tabular}

Values are mean \pm s.e.m., p-values after adjusted for gender, age, hypertension, diabetes, thyroid gland disease, dyslipidemia, smoking, and alcohol consumption (*: within genotype, ${ }^{* *}$ : within gene-diet interaction).

Table 4. Effect of interaction between ADIPOQ polymorphism rs 182052 and dietary intake on fat body mass

\begin{tabular}{|c|c|c|c|c|c|c|}
\hline & & CC $(n=806)$ & CT $(n=1,550)$ & $\mathrm{TT}(\mathrm{n}=743)$ & $p$-value* & $p$-value ${ }^{\star \star}$ \\
\hline \multirow[t]{2}{*}{ Energy, Kcal } & Low & $18.65 \pm 0.29$ & $18.77 \pm 0.20$ & $19.16 \pm 0.30$ & 0.431 & 0.539 \\
\hline & High & $18.64 \pm 0.27$ & $18.83 \pm 0.20$ & $19.72 \pm 0.29$ & 0.016 & \\
\hline \multirow[t]{2}{*}{ Protein, \%E } & Low & $18.44 \pm 0.29$ & $18.67 \pm 0.21$ & $18.97 \pm 0.30$ & 0.452 & 0.437 \\
\hline & High & $18.83 \pm 0.27$ & $18.91 \pm 0.20$ & $19.87 \pm 0.29$ & 0.011 & \\
\hline \multirow[t]{2}{*}{ Fat, \%E } & Low & $18.66 \pm 0.31$ & $18.67 \pm 0.21$ & $19.21 \pm 0.30$ & 0.324 & 0.702 \\
\hline & High & $18.64 \pm 0.27$ & $18.91 \pm 0.20$ & $19.67 \pm 0.29$ & 0.022 & \\
\hline \multirow[t]{2}{*}{ Carbohydrate, \%E } & Low & $18.63 \pm 0.29$ & $18.76 \pm 0.20$ & $19.22 \pm 0.30$ & 0.063 & 0.73 \\
\hline & High & $18.66 \pm 0.28$ & $18.84 \pm 0.20$ & $19.66 \pm 0.29$ & 0.045 & \\
\hline \multirow[t]{2}{*}{ Saturated fatty acid, \%E } & Low & $18.97 \pm 0.29$ & $18.73 \pm 0.21$ & $19.61 \pm 0.30$ & 0.063 & 0.312 \\
\hline & High & $18.37 \pm 0.27$ & $18.86 \pm 0.20$ & $19.30 \pm 0.29$ & 0.045 & \\
\hline \multirow{2}{*}{ Monounsaturated fatty acid, \%E } & Low & $18.78 \pm 0.29$ & $18.77 \pm 0.21$ & $19.60 \pm 0.30$ & 0.063 & 0.743 \\
\hline & High & $18.54 \pm 0.27$ & $18.82 \pm 0.20$ & $19.30 \pm 0.29$ & 0.119 & \\
\hline \multirow[t]{2}{*}{ Polyunsaturated fatty acid, \%E } & Low & $18.62 \pm 0.29$ & $18.73 \pm 0.21$ & $19.09 \pm 0.29$ & 0.488 & 0.399 \\
\hline & High & $18.67 \pm 0.28$ & $18.85 \pm 0.20$ & $19.83 \pm 0.30$ & 0.010 & \\
\hline
\end{tabular}

Values are mean \pm s.e.m., p-values after adjusted for gender, age, hypertension, diabetes, thyroid gland disease, dyslipidemia, smoking, and alcohol consumption (*: within genotype, ${ }^{* *}$ : within gene-diet interaction). 
ary intakes.

On the other hand, recent studies reported that the interaction between genetic variations and obesity, and dietary intakes potentially modulated the risk of obesity as well metabolic syndrome (Chung et al., 2009; Ntalla et al., 2009; Santos et al., 2006; Warodomwichit et al., 2009). In this study, the interaction between different genotypes of ADIPOQ rs182052 and dietary intake on the obesity-related variables such as BMI and fat body mass was analyzed. The intakes of energy, protein, fat, carbohydrate, saturated fatty acid, monounsaturated fatty acid, and polyunsaturated fatty acid were divided according to their respective medians. significant gene-dietary intake interactions on BMl and fat body mass in genotypes of ADIPOQ rs182052 (Table 3 and 4) were not observed, suggesting that the obesity-related variables in women aged between 40-59 years might be more dominantly affected by genotype of ADIPOQ rs182052 than dietary intake.

In conclusion, this study demonstrated that genetic variations in ADIPOQ rs182052 are directly associated with obesity-related variables such as weight, BMI, fat body mass, and waist-hip ratio in Korean women. Moreover, the risk of obesity prevalence was also affected by variations in ADIPOQ rs182052. However, we failed to discover the effects of interaction between gene and dietary intake on obesity. Further studies may be necessary to elucidate the interaction between ADIPOQ gene polymorphism and dietary intakes on obesity-related variables, and confirm our results through measurement of the plasma adiponectin level.

\section{Acknowledgments}

This research was supported by grants from Korea Centers for Disease Control and Prevention, Republic of Korea (4845-301, 4851-302, 4851-307).

\section{References}

Ahn, Y., Kwon, E., Shim, J.E., Park, M.K., Joo, Y., Kimm, K., Park, C., and Kim, D.H. (2007). Validation and reproducibility of food frequency questionnaire for Korean genome epidemiologic study. Eur. J. Clin. Nutr. 61, 1435-1441

Ahn, Y.J., Park, Y.J., Park, S.J., Min, H.S., Kwak, H.K., Oh, K.S., and Park, C. (2007). Dietary Patterns and Prevalence Odds Ratio in Middle-aged Adults of Rural and Mid-size City in Korean Genome Epidemiology Study. Korean J. Nutr. 40, 259-269

Bostrom, M.A., Freedman, B.I., Langefeld, C.D., Liu, L., Hicks, P.J., and Bowden, D.W. (2009). Association of adiponectin gene polymorphisms with type 2 diabetes in an African American population enriched for nephropathy. Diabetes. 58, 499-504.
Cho, Y.S., Go, M.J., Kim, Y.J., Heo, J.Y., Oh, J.H., Ban, H.J., Yoon, D., Lee, M.H., Kim, D.J., Park, M., Cha, S.H., Kim, J.W., Han, B.G., Min, H., Ahn, Y., Park, M.S., Han, H.R., Jang, H.Y., Cho, E.Y., Lee, J.E., Cho, N.H., Shin, C., Park, T., Park, J.W., Lee, J.K., Cardon, L., Clarke, G., McCarthy, M.I., Lee, J.Y., Lee, J.K., Oh, B., and Kim, H.L. (2009). A large-scale genome-wide association study of Asian populations uncovers genetic factors influencing eight quantitative traits. Nat. Genet. 41, 527-534.

Chung, H.K., Chae, J.S., Hyun, Y.J., Paik, J.K., Kim, J.Y., Jang, Y., Kwon, H.M., Song, Y.D., Lee, H.C., and Lee, J.H. (2009). Influence of adiponectin gene polymorphisms on adiponectin level and insulin resistance index in response to dietary intervention in overweight-obese patients with impaired fasting glucose or newly diagnosed type 2 diabetes. Diabetes Care. 32, 552-558.

Cnop, M., Havel, P.J., Utzschneider, K.M., Carr, D.B., Sinha, M.K., Boyko, E.J., Retzlaff, B.M., Knopp, R.H., Brunzell, J.D., and Kahn, S.E. (2003). Relationship of adiponectin to body fat distribution, insulin sensitivity and plasma lipoproteins: evidence for independent roles of age and sex. Diabetologia. 46, 459-469.

Dolley, G., Bertrais, S., Frochot, V., Bebel, J.F., Guerre-Millo, M., Tores, F., Rousseau, F., Hager, J., Basdevant, A., Hercberg, S., Galan, P., Oppert, J.M., Lacorte, J.M., and Clément, K. (2008). Promoter adiponectin polymorphisms and waist/hip ratio variation in a prospective French adults study. Int. .J Obes. (Lond). 32, 669-675.

Drewnowski. A. (2009).Obesity, diets, and social inequalities. Nutr. Rev. 67, S36-39.

Ezzati, M., Martin, H., Skjold, S., Vander, H.S., and Murray, C.J. (2006). Trends in national and state-level obesity in the USA after correction for self-report bias: analysis of health surveys. J. R. Soc. Med. 99, 250-257.

Fawcett, K.A., and Barroso, I. (2010). The genetics of obesity: FTO leads the way. Trends Genet. 26, 266-274

Friedewald, W.T., Levy, R.I., and Fredrickson, D.S. (1972). Estimation of the concentration of low-density lipoprotein cholesterol in plasma without use of the preparative ultracentrifuge. Clin. Chem. 18, 499-502.

Gilardini, L., McTernan, P.G., Girola, A., da Silva, N.F., Alberti, L., Kumar, S., and Invitti, C. (2006). Adiponectin is a candidate marker of metabolic syndrome in obese children and adolescents. Atherosclerosis. 189, 401-407.

Heid, I.M., Henneman, P., Hicks, A., Coassin, S., Winkler, T., Aulchenko, Y.S., Fuchsberger, C., Song, K., Hivert, M.F., Waterworth, D.M., Timpson, N.J., Richards, J.B., Perry, J.R., Tanaka, T., Amin, N., Kollerits, B., Pichler, I., Oostra, B.A., Thorand, B., Frants, R.R., Illig, T., Dupuis, J., Glaser, B., Spector, T., Guralnik, J., Egan, J.M., Florez, J.C., Evans, D.M., Soranzo, N., Bandinelli, S., Carlson, O.D., Frayling, T.M., Burling, K., Smith, G.D., Mooser, V., Ferrucci, L., Meigs, J.B., Vollenweider, P., Dijk, K.W., Pramstaller, P., Kronenberg, F., and van Duijn, C.M. (2010). Clear detection of ADIPOQ locus as the major gene for plasma adiponectin: results of genome-wide association analyses including 4659 European individuals. Atherosclerosis. 208, 412-420. 
Henneman, P., Aulchenko, Y.S., Frants, R.R., Zorkoltseva, I.V., Zillikens, M.C., Frolich, M., Oostra, B.A., van Dijk, K.W., and van Duijn, C.M. (2010). Genetic architecture of plasma adiponectin overlaps with the genetics of metabolic syndrome-related traits. Diabetes Care. 33, 908913.

Kadowaki, T., Yamauchi, T., Kubota, N., Hara, K., and Ueki, K. (2006). Adiponectin and adiponectin receptors in insulin resistance, diabetes, and the metabolic syndrome. J. Clin. Invest 116, 1784-1792.

Korean Nutrition Society. (2006). Can pro 3.0 software. KNS

Li, S., Shin, H.J., Ding, E.L, and van Dam, R.M. (2009). Adiponectin levels and risk of type 2 diabetes: a systematic review and meta-analysis. JAMA 302, 179-188.

Loos, R.J. (2009). Recent progress in the genetics of common obesity. Br. J. Clin. Pharmacol, 68, 811-829.

Hivert, M.F., Manning, A.K., McAteer, J.B., Florez, J.C., Dupuis, J., Fox, C.S., O'Donnell, C.J., Cupples, L.A., and Meigs, J.B. (2008). Common variants in the adiponectin gene (ADIPOQ) associated with plasma adiponectin levels, type 2 diabetes, and diabetes-related quantitative traits: the Framingham Offspring Study. Diabetes 57, 3353-3359.

Ntalla, I., Dedoussis, G., Yannakoulia, M., Smart, M.C., Louizou, E., Sakka, S.D., Papoutsakis, C., and Talmud, P.J. (2009). ADIPOQ gene polymorphism rs1501299 interacts with fibre intake to affect adiponectin concentration in children: the GENe-Diet Attica Investigation on childhood obesity. Eur. J. Nutr. 48, 493-497.

Ong, K.L., Li, M., Tso, A.W., Xu, A., Cherny, S.S., Sham, P.C., Tse, H.F., Lam, T.H., Cheung, B.M., and Lam, K.S. (2010). Association of genetic variants in the adiponectin gene with adiponectin level and hypertension in Hong Kong Chinese. Eur. J. Endocrinol. 163, 251-257.

Richardson, D.K., Schneider, J., Fourcaudot, M.J., Rodriguez, L.M., Arya, R., Dyer, T.D., Almasy, L., Blangero, J., Stern, M.P., Defronzo, R.A., Duggirala, R., and Jenkinson, C.P. (2006). Association between variants in the genes for adiponectin and its receptors with insulin resistance syndrome (IRS)-related phenotypes in Mexican Americans. Diabetologia. 49, 2317-2328.

Santos, J.L., Boutin, P., Verdich, C., Holst, C., Larsen, L.H.,
Toubro, S., Dina, C., Saris, W.H., Blaak, E.E., Hoffstedt, J., Taylor, M.A., Polak, J., Clement, K., Langin, D., Astrup, A., Froguel, P., Pedersen, O., Sorensen, T.I., and Martinez, J.A. (2006). Genotype-by-nutrient interactions assessed in European obese women. A case-only study. Eur. J. Nutr. 45, 454-462.

Scagliusi, F.B., Ferriolli, E., Pfrimer, K., Laureano, C., Cunha, C.S., Gualano, B., Lourenço, B.H., and Lancha, A.H. Jr. (2008). Underreporting of energy intake in Brazilian women varies according to dietary assessment: a cross-sectional study using doubly labeled water. J. Am. Diet. Assoc. 108, 2031-2040.

Swithers, S.E., Martin, A.A., and Davidson, T.L. (2010). High-intensity sweeteners and energy balance. Physiol Behav. 26;100, 55-62.

Walker, C.G., Zariwala, M.G., Holness, M.J., and Sugden, M.C. (2007). Diet, obesity and diabetes: a current update. Clin. Sci. (Lond). 112, 93-111.

Warodomwichit, D., Shen, J., Arnett, D.K., Tsai, M.Y., Kabagambe, E.K., Peacock, J.M., Hixson, J.E., Straka, R.J., Province, M.A., An, P., Lai, C.Q., Parnell, L.D., Borecki, I.B., and Ordovas, J.M. (2009). ADIPOQ polymorphisms, monounsaturated fatty acids, and obesity risk: the GOLDN study. Obesity (Silver Spring). 17, 510517.

Wassel, C.L., Pankow, J.S., Jacobs, D.R.Jr., Steffes, M.W., Li, N., and Schreiner, P.J. (2010). Variants in the Adiponectin Gene and Serum Adiponectin: The Coronary Artery Development in Young Adults (CARDIA) Study. Obesity (Silver Spring). [Epub ahead of print]

Weyer, C., Funahashi, T., Tanaka, S., Hotta, K., Matsuzawa, Y., Pratley, R.E., and Tataranni, P.A. (2001). Hypoadiponectinemia in obesity and type 2 diabetes: close association with insulin resistance and hyperinsulinemia. J. Clin. Endocrinol. Metab. 86, 1930-1935.

World Health Organization. (2000). The Asia- Pacific perspective: Redefining obesity and its treatment. Geneva: WHO. p.18.

World Health Organization. (2006). Obesity and overweight: what are overweight and obesity? http://www.who.int/mediacentre/factsheets/fs311/en/. 